\title{
SOME EXAMPLES OF FINITENESS CONDITIONS IN CENTRE-BY-METABELIAN GROUPS
}

\author{
J. R. J. GROVES \\ (Received 14 November 1983) \\ Communicated by D. E. Taylor
}

\begin{abstract}
Centre-by-metabelian groups with the maximal condition for normal subgroups are exhibited which (a) are residually finite but have quotient groups which are not residually finite; and (b) have all quotients residually finite but are not abelian-by-polycyclic.
\end{abstract}

1980 Mathematics subject classification (Amer. Math. Soc.): 20 F 16, 20 E 26.

Segal, in [1], page 336, observes that the following question is "elusive": does a finitely generated soluble group which has the maximum condition for normal subgroups and is residually finite have all its quotients residually finite?

The purpose of this note is to settle that question by exhibiting some examples of centre-extended-by-metabelian groups.

THEOREM. There exist centre-by-metabelian groups with the maximal condition for normal subgroups which (a) are residually-finite but have non-residually-finite quotients; (b) have all quotients residually-finite but are not abelian-by-polycyclic. The class is parametrised by sequences of integers and the group theoretical properties are related to properties of the sequences of integers.

Let $G_{1}$ be the group

$$
\begin{array}{r}
\left\langle t_{1}, x_{1}, y_{1}, z_{1}^{(i)}:\left[z_{1}^{(i)}, t_{1}\right]=\left[z_{1}^{(i)}, x_{1}\right]=\left[z_{1}^{(i)}, y_{1}\right]=1,\right. \\
\left.\left[x_{1}, y_{1}^{t_{1}^{i}}\right]=z_{1}^{(i)},\left[x_{1}, x_{1}^{t_{1}^{i}}\right]=\left[y_{1}, y_{1}^{t_{1}}\right]=1,(i \in \mathbf{Z})\right\rangle .
\end{array}
$$

- 1985 Australian Mathematical Society $0263-6115 / 85 \$ A 2.00+0.00$ 
Let $N_{1}$ denote the normal closure of $\left\{x_{1}, y_{1}\right\}$ in $G_{1}$ and $Z_{1}$ denote the normal subgroup generated by the $z_{1}^{(i)}$. Then $G_{1} / N_{1}$ is cyclic, $N_{1} / Z_{1}$ is abelian and $Z_{1}$ is a central free abelian group with basis $\left\{z_{1}^{(i)}: i \in \mathbf{Z}\right\}$.

Let $\Gamma=\left\{\gamma_{i}: i \in \mathbf{Z}\right\}$ be a sequence of integers with $\gamma_{0}=1$ and let $H_{\Gamma} \leqslant Z_{1}$ be the subgroup generated by all elements

$$
z_{1}^{(i)}\left(z_{1}^{(0)}\right)^{-\gamma_{i}} \quad(i \in \mathbf{Z}) .
$$

Then $Z_{1} / H_{\Gamma}$ is infinite cylic, generated by the image of $z_{1}^{(0)}=\left[x_{1}, y_{1}\right]$. Let $G(\Gamma)=G_{1} / H_{\Gamma}$ (note that $H_{\Gamma}$ is central and so normal in $G_{1}$ ). We will denote the images of previously defined elements and subgroups in $G(\Gamma)$ by omitting the subscript.

By a bi-monic linear recurrence relation satisfied by $\Gamma$, we mean a relation of the form

$$
\gamma_{i+k}+c_{k-1} \gamma_{i+k-1}+\cdots+c_{1} \gamma_{i+1}+\gamma_{i}=0
$$

holding for all $i \in \mathbf{Z}$ and some $k, c_{j} \in \mathbf{Z}$.

The proof of the theorem involves relating the group-theoretical properties of $G(\Gamma)$ with properties of $\Gamma$. We do this first in a simpler case. For each non-negative integer $n$ denote by $G(\Gamma)_{n}$ the quotient $G(\Gamma) / Z_{n}\left(\right.$ so $G(\Gamma)_{0}=G(\Gamma)$ ).

PROPOSITION 1. For all $n \geqslant 0$ the following two properties are equivalent:

(a) $G(\Gamma)_{n}$ is abelian-by-polycyclic;

(b) $\Gamma$ satisfies a bi-monic linear recurrence relation modulo $n$;

Further, if $n \neq 0$ then these are equivalent to

(c) $G(\Gamma)_{n}$ is residually-finite;

(d) $\Gamma$ is periodic modulo $n$.

Proof. Let $N_{n}$ denote the image of $N$ in $G(\Gamma)_{n}$ and let $C_{n}$ denote the centre of $N_{n}$ in $G(\Gamma)_{n}$. Then (b) holds if and only if

$$
z^{(i+k)} z^{(i+k-1) c_{k-1}} \cdots z^{(i)}=1 \text { in } G(\Gamma)_{n}, \quad \text { for all } i \in \mathbf{Z},
$$

that is, if and only if,

$$
\left[x, y^{t^{i+k}} y^{t^{i+k-1} c_{k-1}} \cdots y^{t^{i}}\right]=1 \text {. }
$$

This, in turn, holds if and only if,

$$
y^{t^{k}} y^{t^{k-1} c_{k-1}} \cdots y^{t} \in C_{n} .
$$

But an expression of this type belongs to $C_{n}$ if and only if $N / C_{n}$ is finitely generated. Hence (b) holds if and only if $N / C_{n}$ is finitely generated. If $N / C_{n}$ is finitely generated, then $G(\Gamma)_{n} / C_{n}$ is polycyclic and so $G\left(\Gamma_{n}\right)$ is abelian-by-polycyclic. 
Conversely, if $G(\Gamma)_{n}$ is abelian-by-polycyclic, then there is an abelian subgroup $D_{n}$ of $N$, normal in $G(\Gamma)_{n}$, with $N / D_{n}$ finitely generated. Thus for some $k, c_{j}$ and all $i$ in $\mathbf{Z}$,

$$
x^{t^{i+k}} x^{t^{i+k-1}} c_{k-1} \ldots x^{t^{t}} \in D_{n} ; y^{t^{i+k}} y^{t^{i+k-1}} c_{k-1} \cdots y^{t^{t}} \in D_{n} .
$$

Using the fact that $D_{n}$ is abelian, it is easily verified that (b) is satisfied.

Suppose now that $n \neq 0$. Then it is clear that (b) and (d) are equivalent. Also, it is well known that (a) implies (c). Assume, then, that $G(\Gamma)_{n}$ is residually finite. Then there is a normal subgroup $T$ of finite index avoiding the finite normal subgroup $Z_{n}$. Thus $G(\Gamma)_{n}$ is a subdirect product of the metabelian group $G(\Gamma)_{n} / Z_{n}$ and the finite group $G(\Gamma)_{n} / T$; in, particular, it is abelian-by-polycyclic.

The next lemma relates the residual finiteness of $G(\Gamma)$ to that of the $G(\Gamma)_{n}$.

LEMMA 2. $G(\Gamma)$ is residually finite if and only if $\left\{n: G(\Gamma)_{n}\right.$ is residually finite $\}$ is infinite.

Proof. Note that, for any infinite set $S$ of positive integers, $Z$ is a subdirect product of $\left\{Z / Z^{n}: n \in S\right\}$ and so $G(\Gamma)$ is a subdirect product of $\left\{G(\Gamma)_{n}\right.$ : $n \in S\}$. Thus, if $\left\{n: G(\Gamma)_{n}\right.$ is residually finite $\}$ is infinite, then $G(\Gamma)$ is a subdirect product of residually finite groups and so residually finite.

For the converse, suppose that $F$ is a normal subgroup of finite index in $G(\Gamma)$. Then, as in the proof of Proposition $1, G(\Gamma) / Z \cap F$ is residually finite. If $\{n$ : $G(\Gamma)_{n}$ is residually finite $\}$ is finite, then $T=\left\{n: Z^{n}=Z \cap F\right.$ for some normal $F$ of finite index $\}$ is finite. Hence the intersection of all the normal subgroups of finite index in $G(\Gamma)$ contains the intersection of all $Z^{n}(n \in T)$ which is non-trivial. Thus $G(\Gamma)$ is not residually finite.

We can summarise this as follows.

COROllaRY 3. $G(\Gamma)$ is a finitely generated centre-by-metabelian group with the maximal condition on normal subgroups.

(a) $G(\Gamma)$ is residually finite if and only if $\Gamma$ is periodic modulo $n$ for infinitely many distinct $n$.

(b) Every quotient of $G(\Gamma)$ is residually finite if and only if $\Gamma$ is periodic modulo $n$ for all $n$.

(c) $G(\Gamma)$ is abelian-by-polycyclic if and only if $\Gamma$ satisfies a bi-monic linear recurrence relation.

Proof. A combination of Proposition 1 and Lemma 2 proves all but the "if" implication of (b). Suppose, then, that $\Gamma$ is periodic modulo $n$ for all $n$. By 
Proposition $1, G(\Gamma)_{n}$, together with all of its quotients, is residually finite for all $n$. But any monolithic quotient of $G(\Gamma)$ must have a finite centre and so be a quotient of some $G(\Gamma)_{n}$. Thus each monolithic quotient of $G(\Gamma)$ is residually finite and so $G(\Gamma)$ is residually finite.

Finally, to complete the proof of the Theorem, we must distinguish the three properties of sequences mentioned in the Corollary.

LemMa 4. (a) There exists a sequence $\Gamma$ which is periodic modulo $n$ for infinitely many, but not for all, $n$; (b) there exists a sequence $\Gamma$ which is periodic modulo $n$ for all $n$ but which satisfies no bi-monic linear recurrence relation.

This is surely well-known and elementary but inelegant proofs are not difficult to find. I do not, however, have the short and elegant proof which it seems likely must exist. I therefore leave to the interested reader the (hopefully enjoyable) task of finding a suitable agrument.

Combining Corollary 3 with Proposition 4, the proof of the Theorem is complete.

\section{References}

[1] D. Segal, 'On the residual simplicity of certain modules', Proc. London Math. Soc. (3) 34 (1977), 327-353.

Department of Mathematics

University of Melbourne

Parkville, Victoria 3053

Australia 